\title{
SUSTENTABILIDADE NA PRODUÇÃO DE REFEIÇÕES: UMA REVISÃO DAS EVIDÊNCIAS CIENTÍFICAS
}

\section{SUSTAINABILITY IN THE PRODUCTION OF MEALS: A REVIEW OF SCIENTIFIC EVIDENCE}

\author{
Júlia Ferreira Yano' \\ ${ }_{1}$ Pontifícia Universidade Católica de Goiás. Escola de Ciências Sociais e da Saúde. Goiânia, \\ Goiás, Brasil. \\ https://orcid.org/0000-0003-3429-8370 \\ julia_yano@hotmail.com \\ Profa. Orientadora Me. Nair Augusta de Araújo Almeida Gomes ${ }^{1}$ \\ ${ }_{1}$ Pontifícia Universidade Católica de Goiás. Escola de Ciências Sociais e da Saúde. Goiânia, \\ Goiás, Brasil. \\ https://orcid.org/0000-0001-6882-0388 \\ nairaugustaalmeida@yahoo.com.br
}

\begin{abstract}
1 Participação na formulação e elaboração do artigo. Desenho do Projeto, Escrita - Primeira Redação, Metodologia, Discussão dos Resultados, Escrita - Revisão e aprovação da versão final. ${ }^{2}$ Participação na formulação e elaboração do artigo. Desenho do Projeto, Escrita - Primeira Redação, Metodologia, Discussão dos Resultados, Escrita - Revisão e aprovação da versão final do trabalho.
\end{abstract}

\section{RESUMO}

Hoje em dia, há uma grande preocupação com dois problemas que atingem o nosso planeta, o desperdício de alimentos, enquanto pessoas passam fome, e o esgotamento dos recursos naturais. Este trabalho teve por objetivo aprofundar os conhecimentos acerca das possibilidades de 0 profissional nutricionista atuar de forma sustentável na produção de refeições. Para tanto foi feita uma revisão da literatura no período de 2004 a 2020. Foram observadas perdas de alimentos desde a produção até o consumo final e, portanto, a necessidade de um consumo consciente, propiciando assim, o acesso ao alimento por pessoas em estado de insegurança alimentar. Ao ser discutido o aproveitamento integral dos alimentos, prática sustentável e ecologicamente correta, verificou-se que essa proporciona uma maior utilização dos recursos naturais, diminuição dos gastos com a alimentação, diversificando os hábitos alimentares e melhorando o valor nutricional das preparações. Além de contribuir na redução da incidência de carências nutricionais ou doenças advindas de uma baixa ingestão alimentar. Pode-se constatar que as Unidades de Alimentação e Nutrição (UAN) são passíveis de executar ações, durante todo o processo produtivo, que promovam o desenvolvimento sustentável. Conclui-se que o nutricionista tem papel fundamental na implantação e manutenção da sustentabilidade dentro das UAN, mediante sua prática, na produção das refeições, orientação de funcionários e clientes, bem como a responsabilidade social e ambiental. Estudos dessa natureza são importantes por apresentar as práticas adotadas no segmento de produção de refeições e para estimular investigações futuras que sinalizem para a redução dos impactos ambientais nessa atividade.

Palavras-chave: Desenvolvimento Sustentável. Desperdício de alimentos. Serviços de Alimentação. 


\section{ABSTRACT}

Nowadays, two critical problems affect our planet, waste, while people go hungry and the depletion of natural resources. This work intends to present the possibilities of a nutritionist applicate sustainable actions in the production of meals. To this end, it was done a study reviewing publications from 2004 to 2020. Food losses were observed from production to final consumption and, therefore, the need for conscious consumption, thus providing access to food for people in a state of food insecurity. When discussing the full utilization food product, a sustainable and ecologically practice, it was found that it provides greater use of natural resources, decreased spending on food, diversifying eating habits and improving the nutritional value of preparations. In addition, it contributes to reduce the incidence of nutritional deficiencies or diseases resulting from low food intake. The Food and Nutrition Units (UAN) can adopt practices in the production of meals that promote sustainable development. It is concluded that the nutritionist has a fundamental role in the implementation and maintenance of sustainability within the Food and Nutrition Units, through the actions in aspects related to production, employees, and customers, as well as social and environmental responsibility. Evaluative studies of this nature are important to contribute to the diagnosis of reality in meal production segment and to promote further investigations that show the reduction of environmental impacts on this activity.

Keywords: Sustainable Development. Food Wastefulness. Food Services.

\section{INTRODUÇÃO}

Segundo dados da Organização das Nações Unidas para a Alimentação e a Agricultura (FAO), a cada ano, um terço dos alimentos produzidos para consumo humano é perdido ou desperdiçado globalmente (FAO, 2011). Os números do desperdício impressionam (Quadro 1).

Quadro 1- Desperdício de Alimentos no Mundo, na América Latina e no Brasil.

\begin{tabular}{|l|c|c|}
\hline \multirow{2}{*}{ Local } & \multicolumn{2}{|c|}{ Quantidade } \\
\cline { 2 - 3 } & $\begin{array}{c}\text { Toneladas de } \\
\text { alimentos/ ano }\end{array}$ & $\begin{array}{c}\text { Suficiente } \\
\text { para } \\
\text { alimentar }\end{array}$ \\
\hline Mundo & 1,3 bilhão & $\begin{array}{c}2 \text { bilhões } \\
\text { de } \\
\text { pessoas }\end{array}$ \\
\hline $\begin{array}{l}\text { América } \\
\text { Latina }\end{array}$ & 127 milhões & $\begin{array}{c}36 \\
\text { milhões } \\
\text { de } \\
\text { pessoas }\end{array}$ \\
\hline Brasil & 26 milhões & $\begin{array}{c}13 \\
\text { milhões } \\
\text { de } \\
\text { pessoas }\end{array}$ \\
\hline
\end{tabular}

Fonte: FAO, 2018; PORPINO et al.,2018 e CEDES, 2018.

Enquanto toneladas de alimentos são descartadas intencionalmente nos últimos estágios da cadeia produtiva, devido ao comportamento adotado no comércio varejista, nos restaurantes e domicílios, milhões de pessoas se encontram em condição de insegurança alimentar.

Estima-se que no Brasil, em média, 70 mil toneladas de alimentos são descartadas diariamente, o que leva a nação a ser conhecida como o país do desperdício (BADAWI, 2020). Segundo dados de pesquisa realizada pela Empresa Brasileira de Pesquisa Agropecuária (EMBRAPA), o desperdício de alimentos ocorre principalmente devido a fatores comportamentais, conforme apresentado na tabela 1. Essa prática gera um impacto 
negativo no meio ambiente e na saúde das pessoas (SANTOS, 2008).

Tabela 1 - Principais fatores comportamentais que contribuem para o alto desperdício de alimentos no Brasil.

\begin{tabular}{|c|c|}
\hline Fatores de desperdício & $\begin{array}{l}\text { Percentual de } \\
\text { respostas }\end{array}$ \\
\hline $\begin{array}{l}\text { Preferência por comida } \\
\text { fresca à mesa }\end{array}$ & $77 \%$ \\
\hline $\begin{array}{l}\text { Compras de alimentos em } \\
\text { grandes quantidades, para } \\
\text { manter a despensa } \\
\text { abastecida }\end{array}$ & $68 \%$ \\
\hline $\begin{array}{l}\text { Indiferença a respeito da } \\
\text { quantidade de comida } \\
\text { disponível na despensa ou } \\
\text { na mesa }\end{array}$ & $59 \%$ \\
\hline $\begin{array}{l}\text { Preparo de alimentos mais } \\
\text { de uma vez ao dia ("é melhor } \\
\text { sobrar que faltar") }\end{array}$ & $56 \%$ \\
\hline
\end{tabular}

Estão aumentando os debates sobre as questões ambientais e a preocupação crescente acerca do esgotamento dos recursos naturais, requerendo mudanças de comportamentos e nas formas de agir e de pensar. No caso dos alimentos, é inconcebível descartar partes nutritivas, enquanto parcela significativa da população sofre com desnutrição e fome (CARDOSO et al., 2015).

Nas últimas décadas houve mudanças nos hábitos e no padrão de consumo alimentar dos indivíduos, que passaram a realizar parte das refeições fora dos domicílios (AKUTSU et al., 2005). Tal prática impacta no meio ambiente e interfere na sustentabilidade. Pois, no decorrer do processo produtivo há gasto de água e energia, geração acentuada de resíduos sólidos, em decorrência do planejamento ineficiente de cardápios e das compras, recursos humanos sem qualificação, entre outros (BARTHICHOTO et al., 2013).

Por serem as Unidades de Alimentação e Nutrição (UAN) um dos ambientes de trabalho do nutricionista, o tema Educação Ambiental deve ser amplamente abordado na formação desse profissional. A fim de que, por meio de práticas ecologicamente corretas, possa contribuir para o uso sustentável dos recursos naturais, no controle e na redução da produção de resíduos (SAURIM; BASSO, 2008; GONÇALVES et al., 2018).

Desta forma justifica-se a realização de um estudo que venha a aprofundar os conhecimentos acerca das possibilidades de o profissional nutricionista atuar de forma sustentável na produção de refeições.

\section{METODOLOGIA}

Para o alcance dos objetivos propostos, foi realizada uma revisão narrativa da literatura. Nessa perspectiva, o levantamento dos artigos foi realizado nas bases de dados La Literatura Latino Americana e do Caribe em Ciências da Saúde (Lilacs), Scientific Eletronic Library Online (SCielo) e Sistema de Legislação em Saúde, a partir da utilização de palavras-chaves: aproveitamento integral dos alimentos, desperdício de alimentos, gestão ambiental, 
unidades de alimentação e nutrição, nutricionista, sustentabilidade, nutrição e sustentabilidade, desenvolvimento sustentável. Também foram analisados artigos e materiais técnicos que se encontram nas referências bibliográficas das fontes indexadas.

O estudo buscou enfatizar ações sustentáveis do profissional nutricionista dentro de uma Unidade de Alimentação e Nutrição, juntamente com o aproveitamento integral dos alimentos voltados para redução do desperdício e manutenção da saúde humana.

Os critérios de inclusão, documentos nos idiomas português e inglês, que abordassem o tema em questão, publicados no período de 2004 a 2020, disponíveis e gratuitos. Excluíram-se em função da dubiedade das fontes e/ou irrelevância do artigo para a construção da pesquisa, artigos não disponibilizados na íntegra, ou que não se enquadraram nos objetivos do presente estudo analisando-se inicialmente título e resumo.

De posse dos artigos e materiais selecionados, procedeu-se à leitura minuciosa e integral de cada documento. Foram selecionados três temas chaves para nortear a construção textual, a saber: desperdício de alimentos; aproveitamento integral dos alimentos e o papel do nutricionista em uma UAN.

\section{REVISÃO DE LITERATURA}

\subsection{Desperdício de alimentos}

No mundo, um terço de toda a produção de alimentos se torna resíduo alimentar. Deste quantitativo $45 \%$ são referentes a frutas e legumes, $35 \%$ peixes e frutos do mar, $30 \%$ cereais, $20 \%$ produtos lácteos e $20 \%$ carne (CEDES, 2018).

Segundo a FAO, essa situação contribui para a insegurança alimentar global, bem como, agrava os impactos ambientais e o uso de recursos das cadeias alimentares (FAO, 2013).

Para Peixoto e Pinto (2016), a perda de alimentos é a redução involuntária da disponibilidade destes para o consumo humano, resultante de ineficiências nas cadeias produtivas, tais como deficiências de infraestrutura e logística, tecnologias de produção obsoletas ou pouco eficientes, baixa capacidade gerencial ou de habilidades técnicas das pessoas.

Já o desperdício de alimentos é o descarte intencional de produtos alimentares realizado por varejistas e consumidores. Nas Unidades de Alimentação e Nutrição (UAN), o desperdício pode ser mensurado a partir das sobras de alimentos (alimentos que são preparados e não consumidos) e dos restos (alimentos servidos e não consumidos) (ZANDONADI; MAURÍCIO, 2012). Segundo os autores, o planejamento inadequado do número de refeições, per capita inadequado e preferências alimentares são fatores que 
influenciam o desperdício (ZANDONADI; MAURÍCIO, 2012).

Com o intuito de contribuir para reduzir as perdas e desperdícios de alimentos, diversos países, por meio de políticas e programas, estabelecem ações para identificar possíveis soluções, visando corrigir os erros de todas as etapas da cadeia produtiva (CEDES, 2018).

No Brasil, o desperdício ocorre na maioria das vezes, devido ao desconhecimento por parte dos consumidores acerca dos princípios nutritivos dos alimentos, bem como, por inadequações quanto ao aproveitamento, forma de preparo, armazenamento e refrigeração, e ainda pela falta de planejamento de compras (INSTITUTO AKATU, 2004; GONDIM et al., 2005).

Como consequência às perdas e ao desperdício de alimentos ocorridas em todo o planeta, uma grande quantidade de água doce é perdida e/ou contaminada devido ao descarte incorreto dos resíduos; as terras agricultáveis são degradadas, desgastadas e contaminadas; há desperdício de energia do plantio ao consumo, e emissão de gases de efeito estufa na atmosfera (CEDES, 2018).

Esse cenário resulta em uma menor quantidade de alimentos disponíveis para comercialização, ademais, impacta nos preços, dificultando o acesso por parte do consumidor (FAO, 2008).

Há também os impactos sociais, ou seja, práticas de desperdício e, por conseguinte, a insegurança alimentar, questões de dimensão humana, ética e moral (GALLO, 2018). A redução das perdas e desperdícios de alimentos, portanto, é fundamental para reduzir os impactos sociais, econômicos e ambientais no planeta.

Como medida de ação à segurança alimentar e ao desperdício surgiram os Bancos de Alimentos. Estes têm por propósito a arrecadação de doações de alimentos que seriam posteriormente descartados, embora próprios ao consumo humano quanto aos aspectos nutricionais e higiênicos sanitários (MDS, 2015).

Além dos Bancos de Alimentos, outra proposta para minimizar o desperdício é o Aproveitamento Integral de Alimentos (AIA). Estudos apontam que partes não usualmente consumidas como talos, folhas, cascas e sementes de alimentos são, muitas vezes, mais nutritivas que as consumidas (LIMA et al., 2008; MONTEIRO, 2009).

O mundo reconheceu o problema e a Cúpula das Nações inseriu como um dos Objetivos de Desenvolvimento Sustentável (ODS), o objetivo 12, assegurar padrões de produção e de consumo sustentáveis, e cuja meta 12.3 objetiva, até 2030 , reduzir pela metade o desperdício de alimentos per capita mundial, nos níveis de varejo e do consumidor, e reduzir as perdas de alimentos ao longo das cadeias de produção e abastecimento, incluindo as perdas póscolheita (ONUBR, 2018). 
As perdas e os desperdícios de alimentos são indicativos da ineficiência dos nossos sistemas alimentares. A atuação do profissional nutricionista, de maneira constante, por meio de um bom planejamento, ações de educação alimentar e nutricional junto aos comensais e colaboradores, sobre o AIA, sensibilização em relação a redução dos níveis de desperdício, monitoramento e treinamento dos colaboradores são estratégias que irão contribuir para a utilização dos recursos naturais de maneira consciente e ambientalmente sustentável.

\subsection{Aproveitamento integral dos alimentos}

Alimentação é a base da vida e dela depende o estado de saúde do ser humano. O desconhecimento dos princípios nutritivos e o não aproveitamento de todas as partes do alimento resultam no desperdício de toneladas de recursos alimentares (BARBOSA, 2018). Por outro lado, a utilização deles na totalidade promove uma melhor qualidade na alimentação e reduz a produção do lixo orgânico (GOMES; TEIXEIRA, 2017).

O aproveitamento integral dos alimentos (AIA), consiste na utilização de partes do alimento que geralmente são descartadas, a exemplo das cascas, entrecascas, sementes, folhas e talos. Essa prática proporciona diminuição dos gastos com a alimentação da família, diversificação dos hábitos alimentares e melhora no valor nutricional das preparações (SANTANA; OLIVEIRA, 2005; GONDIM et al., 2005).

Além de contribuir na redução da incidência de carências nutricionais, como a desnutrição, obesidade ou doenças advindas de uma baixa qualidade na ingestão alimentar (MONTEIRO, 2009).

Embora não seja uma prática muito utilizada pela população, as receitas que utilizam o alimento em sua totalidade são bem aceitas nos diferentes estratos socioeconômicos. Nutricionalmente, possibilita o consumo de nutrientes como as vitaminas e os sais minerais que auxiliam em tratamentos e prevenções de doenças (VIERA et al., 2010; DAMINANI et al., 2011).

O AIA permite ainda, que sobrem produtos como verduras, legumes e frutas no mercado e, assim, pela lei da oferta e da procura, os valores destes itens tendem a reduzir (DAMINANI et al, 2011). E como consequência, possibilita 0 acesso às pessoas de menor poder aquisitivo.

Criar receitas utilizando o alimento em sua totalidade vai para além de evitar o desperdício, significa reciclar, respeitar e alimentar-se bem com prazer e dignidade (BADAWI, 2020), bem como, contribuir para diminuição dos impactos ambientais (CARDOSO et al., 2015).

No Brasil, muitos ainda se mostram resistentes à prática do $A I A$, isso porque nosso comportamento alimentar é guiado pelos fatores sociais e psicológicos, como 
crenças e culturas (DANIEL; GHISLENI, 2016).

Na América Latina tem-se o hábito de valorizar a mesa farta e comida fresca, comportamentos que levam as famílias a terem um estoque abundante de comida em casa. Isso gera o ciclo do desperdício, caracterizado por compras abundantes, grande estoque alimentício e alto descarte de restos de comida (PORPINO et al., 2018).

Dados de pesquisas realizadas pelo Instituto Akatu e Programa de Orientação Alimentar (POA) do Banco de Alimentos evidenciam $O$ desconhecimento pelo consumidor sobre o AIA (INSTITUTO AKATU, 2004; BANCO DE ALIMENTOS, 2018).

A prática do AIA deve ser apresentada e discutida pelo profissional nutricionista de forma que as pessoas tenham informações sobre os benefícios, os princípios nutritivos das partes dos alimentos normalmente descartadas, e as possibilidades de utilização destas no preparo de refeições nutritivas e palatáveis. A fim de que todos possam aproveitar os benefícios do consumo dos alimentos in natura.

Utilizar o alimento na sua totalidade é uma forma de evitar desperdícios, melhorar nutricionalmente a alimentação, reduzir os impactos ambientais e diminuir o preço dos alimentos (CARDOSO et al., 2015).

\subsection{Ações sustentáveis em uma unidade de alimentação e nutrição}

O termo sustentabilidade implica em atender às necessidades presentes sem comprometer às necessidades futuras (BARTHICHOTO, 2013), visto que, a natureza não é uma fonte ilimitada de recursos e suas reservas devem ser utilizadas de forma racional e comedida (ROOS; BECKER, 2012).

A sustentabilidade abrange as dimensões ambiental, econômica, social e da saúde. Sobre o sistema alimentar, tal prática consiste em utilizar a matéria-prima e os recursos naturais de forma consciente, numa postura que preserva a biodiversidade do ecossistema que os produzem (PIMENTA; GOMES, 2012).

As UAN são unidades que pertencem ao setor de alimentação coletiva, com a finalidade de administrar a produção de refeições nutritivas e com um bom padrão higienicossanitário, a fim de contribuir para a saúde da coletividade e para o desenvolvimento de hábitos alimentares saudáveis (COLARES; FREITAS, 2007).

As UAN podem gerar grande impacto no meio ambiente e na saúde da população. Diante desse fato, compete ao gestor das unidades, analisar os problemas ambientais e aplicar os princípios da sustentabilidade, bem como, ao nutricionista, por se reconhecer um profissional consciente dos seus deveres e de suas possibilidades de contribuir para a sociedade e o meio ambiente (VEIROS; PROENÇA, 2010; ROOS; BECKER, 2012).

O profissional da nutrição que desempenha seu papel de forma sustentável 
gerencia adequadamente os resíduos sólidos, considerando as questões que permeiam essa prática, procura alternativas visando a preservação do meio ambiente e otimiza o uso das matérias-primas (MARTINEZ, 2012; POSPISCHEK et al., 2014; ALMEIDA; SANTANA; MENEZES, 2015).

O desperdício nas UAN pode ocorrer na etapa de aquisição dos gêneros alimentícios, na produção e no consumo das refeições (SILVÉRIO; OLTRAMARI, 2014). $\mathrm{Na}$ sua rotina de trabalho, o nutricionista executa várias atividades a fim de reduzir o desperdício e melhorar a qualidade dos alimentos, adotando estratégias de gerenciamento ambiental que visam minimizar impactos e maximizar a produtividade (ALEVATO; ARAÚJO, 2009; CAMPOS; IKEDA; SPINELLI, 2012).

Dentre as atividades realizadas destacam-se o armazenamento adequado dos gêneros alimentícios, o pré-preparo monitorado, planejamento do cardápio e parceria com os fornecedores para adquirir alimentos de qualidade (SILVÉRIO; OLTROMARI, 2014).

Com esse propósito, o nutricionista pode realizar de forma contínua a padronização de receitas, estabelecer os per capita, avaliar diariamente as sobras e acompanhar o número diário de comensais (TEIXEIRA et al., 2017).

As ações de controle de desperdício permitem a detecção de práticas que geram o aumento dos gastos e a implementação das ações corretivas com vistas a erradicar a curto, médio e longo prazo, de acordo com a realidade de cada UAN (ZANDONADI; MAURÍCIO, 2012; MARQUES; COELHO; HORTS, 2008).

Para Rodrigues et al. (2012), devem ser adotadas posturas a favor da preservação do meio ambiente, por meio do uso adequado da água, produção e destino de resíduos sólidos, coleta seletiva de lixo, descarte adequado do óleo, entre outros. Tais questões são um desafio para os gestores que devem controlar os custos, e atender as demandas e necessidades dos clientes (SPINELLI, 2009).

Uma alternativa ambientalmente sustentável para a utilização dos resíduos orgânicos seria a compostagem, e posterior utilização desse composto orgânico nas hortas ou jardinagem.

Segundo Abreu, Spinelli e Pinto (2013), as ações contra o desperdício nos setores de produção de refeições devem abranger a gestão dos recursos humanos, de materiais e da produção. A gestão dos colaboradores se dará por meio de treinamento nas etapas de recebimento, armazenamento, pré-preparo, preparo e distribuição das refeições. $\mathrm{Na}$ gestão de materiais, destacam-se a seleção dos fornecedores, armazenamento e controle adequados dos produtos, utilização de utensílios e equipamentos adequados à produção das refeições e realização de coleta seletiva dos materiais a serem reciclados. $\mathrm{Na}$ 
gestão de produção devem ser observados dentre outros a ficha técnica de preparo e o critério tempo/temperatura no preparo dos alimentos.

Para minimizar o desperdício de água, orienta-se a capacitação dos funcionários sobre o uso racional da água, manutenção dos equipamentos e correção de vazamentos na rede de água, utilização de materiais de limpeza biodegradáveis e a adoção de estratégias de reaproveitamento e reuso de água, quando possível (VALLE, 2006).

Harmon e Gerald (2007) apresentam como práticas sustentáveis em uma UAN o consumo de alimentos frescos ou minimamente processados, orgânicos, produzidos localmente, provenientes da agricultura familiar, o consumo de proteína de fontes vegetais, a compra de alimentos a granel visando a redução dos resíduos de embalagens, a compostagem, a reciclagem e o incentivo a hortas escolares, domiciliares e urbanas.

Para um processo sustentável de produção de refeições, requer-se 0 treinamento dos colaboradores, que a partir das informações e conhecimentos partilhados poderão adotar por práticas o uso racional da água, energia e demais recursos (SPINELLI, 2009).

Enfim, percebe-se que os problemas decorrentes da geração de resíduos sólidos e desperdício de alimentos em UAN é um problema de gestão desses estabelecimentos.

Cabe ao profissional nutricionista considerar a dimensão ambiental no planejamento das atividades necessárias para a produção e distribuição de refeições, a fim de minimizar os impactos ambientais decorrentes dessa atividade.

Teixeira et al. (2006), Mezomo (2002) e Abreu; Spinelli; Pinto (2013), reiteram que, para o nutricionista atuar de forma sustentável na produção de refeições, é importante a realização de um planejamento efetivo considerando os seguintes fatores: a quantidade de refeições a ser preparada, o número de comensais, o cardápio diário, a estação climática, a sazonalidade da produção de hortifrutigranjeiros, a política de compra dos produtos pelas empresas, as características dos alimentos que influenciam no tempo e forma de armazenamento, o consumo de água e de energia na preparação dos alimentos, a utilização de produtos préprontos, a quantidade de sobras, a geração de resíduos e de desperdícios, entre outros fatores.

\section{CONCLUSÃO}

Ao ser discutido o desperdício, foi abordado o quanto se perde em alimentos ao longo da cadeia de produção e, portanto, a necessidade do consumo consciente, possibilitando o acesso à alimentação por pessoas em estado de insegurança alimentar. 
Já a sustentabilidade, é de fundamental importância, para a garantia da vida da geração atual e das gerações futuras, a compreensão acerca da necessidade de conservar os recursos naturais e adotar condutas que respeitem a capacidade de produção e de renovação do nosso planeta.

Sobre a prática do aproveitamento integral dos alimentos, sob a perspectiva da segurança alimentar, desperdício e sustentabilidade, no Brasil, a situação de insegurança alimentar coexiste com a cultura do desperdício e muitos ainda são resistentes em aproveitar os alimentos na sua totalidade.

Conclui-se que o nutricionista tem papel fundamental na implantação e manutenção da sustentabilidade dentro das UANs, mediante atuação em aspectos relacionados à produção das refeições, atitudes dos funcionários e clientes, e utilização de recursos, pautada na responsabilidade social e ambiental.

\section{REFÊRENCIAS}

ABREU, E.S.; SPPINELLI, M.G.N; PINTO, A.M.S. Gestão de unidades de alimentação e nutrição: um modelo de fazer. 5. ed. São Paulo: Metha, 2013. 378p.

AKUTSU, R.C.; BOTELHO, R.A.; CAMARGO, E.B.; SÁVIO, K.E.O.; ARAÚJO, W.C. A ficha técnica de preparação como instrumento de qualidade na produção de refeições. Revista de Nutrição, v. 18, n. 2, p.277-9, 2005.

ALEVATO, H.; ARAÚJO, E.M.G. Gestão, organização e condições de trabalho. In: CONGRESSO NACIONAL DE EXCELÊNCIA EM GESTÃO, 5., 2009, Niterói.
ALMEIDA, J.L.; SANTANA, K.B.; MENEZES, M.B.C. Sustentabilidade em unidades de alimentação e nutrição. Revista Encontro Internacional de Formação de Professores e Fórum Permanente de Inovação Educacional, v.8, n.1, p.1-15, 2015.

BADAWI, C. Aproveitamento Integral dos Alimentos: Melhor Sobrar do que faltar? São Paulo. Disponível em: http: //www.nutrociencia.com.br. Acesso em: 20 abr.2020.

BANCO DE ALIMENTOS - ONG. Relatório de atividades 2018.2 Disponível em:<https://www.bancodealimentos.org.br/wp -content/uploads/2019/04/0326relatoriooba2018 textorevisado_design_fimquad 20190416.pdf>. Acesso em: 22 abr. 2020.

BARBOSA, T.J. A importância e o uso de talos, cascas, semente $e$ folhas na alimentação familiar. 2018. Petrolina: Universidade Federal do Vale do São Francisco, 2018. 27 p.

BARTHICHOTO, M.; MATIAS, A.C.G.; SPINELLI, M.G.N.; ABREU, E.S. Responsabilidade Ambiental: Perfil das Práticas de Sustentabilidade desenvolvidas em Unidades Produtoras de Refeições do bairro de Higienópolis, Município de São Paulo. Revista Eletrônica Qualit@s, v.14, n.1, p.1-9, 2013.

CAMPOS, J.R.; IKEDA, V.; SPINELLI, M.G.N. Otimização de espaço físico em Unidade de Alimentação e Nutrição (UAN) considerando avanços tecnológicos no segmento de equipamentos. Revista Univap, v.18, n.32, p.31-41, 2012.

CARDOSO, F.T.; FRÓES, S.C.; FRIEDE, R.; MORAGAS, C.J.; MIRANDA, M.G.; AVELAR, K.E.S. Aproveitamento integral de Alimentos e o seu impacto na Saúde. Sustentabilidade em Debate, v.6, n.3, p.131-143, 2015.

CENTRO DE ESTUDOS E DEBATES ESTRATÉGICOS (CEDES). Consultoria Legislativa da Câmara dos Deputados. Perdas e desperdício de alimentos - 
estratégias para redução. Série de cadernos de trabalhos e debates 3. Brasília: DF, 2018. $260 p$.

COLARES, L.G.T.; FREITAS, C.M.P. Processo de trabalho e saúde de trabalhadores de uma unidade de alimentação e nutrição: entre a prescrição e o real do trabalho. Cadernos de Saúde Pública, v.23, n.12, p.3011-3020, 2007.

DAMIANI, C.; SILVA, F.A.; RODOVALHO, E.C.; BECKER, F.S.; ASQUIERI, E.R.; OLIVEIRA, R.A.; LAGE, M.E. Aproveitamento de resíduos vegetais para produção de farofa temperada. Revista Alimentos e Nutrição, v.22, n.4, p.657-662, 2011.

DANIEL, B.S.; GHISLENI, C.P. Desenvolvimento de um produto alimentício com aproveitamento integral do alimento. Revista da Associação Brasileira de Nutrição, ano 7, n.2, p.43-49, 2016.

FOOD AND
AGRICULTURE
ORGANIZATION OF THE UNITED
NATIONS. Corporate Document Repository. Crop Prospects and Food Situation n. 4. Rome: FAO, 2008.

\section{FOOD AND AGRICULTURE} ORGANIZATION OF THE UNITED NATIONS. Global food losses and food waste - extent, causes and prevention. iniciativas brasileiras. Rome: FAO, 2011. 29p.

\begin{tabular}{lll} 
FOOD AND & \multicolumn{2}{c}{ AGRICULTURE } \\
ORGANIZATION OF THE UNITED
\end{tabular}
NATIONS. Food wastage footprint Impacts on natural resources. Rome: FAO, 2013. 61p.

FOOD AND AGRICULTURE ORGANIZATION OF THE UNITED NATIONS. Building climate resilience for food security and nutrition. Rome: FAO, 2018. 202p.

GALLO, Juliana Maria Altavista Sagretti. Avaliação da percepção de participantes do Banco de Alimentos da Companhia de Entrepostos e Armazéns Gerais de São
Paulo sobre o processo de irradiação de alimento. Divulgação do tratamento de alimentos por radiação ionizante: desenvolvimento de questionário e análise sensorial. 2018. Tese (doutorado) - Instituto de Pesquisas Energéticas e Nucleares, Autarquia associada a Universidade de São Paulo, São Paulo, 2018. 245 p.

GOMES, M.E.M.; TEIXEIRA, C. Aproveitamento Integral dos Alimentos: Qualidade Nutricional e Consequência Ambiental no Ambiente Escolar. Ensino, Saúde e Ambiente, v.10, n.1, p. 203- 217, 2017.

GONÇALVES, L.C.; SILVEIRA, C.S.; PEREIRA, M.C.; HELBIG, E. Sustentabilidade ambiental em restaurantes comerciais da Zona Central de Pelotas - RS. Revista Gestão \& Sustentabilidade Ambiental, v.7, n.2, p.525-539, 2018.

GONDIM, J.A.M.; MOURA, M F.V.; DANTAS, A.S.; MEDEIROS, R.L.; SANTOS, K. M. Composição centesimal e de minerais em cascas de frutas. Ciência e Tecnologia de Alimentos, v.25, n.4, p. 825-27, 2005.

HARMON, A.H., GERALD, B.L. Position of the American Dietetic Association: Food and Nutrition Professionals can implement practices to conserve natural resource and support ecological sustainability. Journal of the American Dietetic Association, v.107, n. 6, p.1033-43, 2007.

\section{INSTITUTO AKATU. Um panorama} brasileiro da alimentação. Caderno Temático: a nutrição e o consumo consciente. São Paulo, 2004.

LIMA, G.P.P. et al. Parâmetros bioquímicos em partes descartadas de vegetais. In: PROGRAMA ALIMENTE-SE BEM: tabela de composição química das partes não convencionais dos alimentos. São Paulo: SESI, 2008.

MARQUES, E.S.; COELHO, A.I.M.; HORTS, $S$. Controle de sobra limpa no processo de produção de refeições em restaurantes. 
Revista Higiene Alimentar, v.22, n.160, p.20-24, 2008.

MARTÍNEZ, Monnya Karollyne da Silva. Gastronomia e sustentabilidade: um estudo de legislação ambiental e sanitária nos restaurantes de Macapá. 2012. Dissertação (Mestrado em Direito Ambiental e Políticas Públicas) - Universidade Federal do Amapá, 2012.

MEZOMO, I. de B. Os serviços de alimentação: planejamento e administração. São Paulo: Manole, 2002.

MINISTÉRIO DO DESENVOLVIMENTO SOCIAL (MDS). Serviço Social do Comércio SESC Mesa Brasil. Fundação Gaúcha dos Bancos Sociais. Rede de Bancos de Alimentos do Rio Grande do Sul. Rede Brasileira de Bancos de Alimentos. 2015. $22 \mathrm{f}$.

MONTEIRO, Betânia de Andrade. Valor nutricional de partes convencionais e não convencionais de frutas e hortaliças. 2009. Dissertação (Mestrado em Energia na Agricultura) - Universidade Estadual Paulista "Júlio de Mesquita Filho", Faculdade de Ciências Agronômicas - UNESP, Botucatu, 2009.

NAÇÕES UNIDAS NO BRASIL (ONUBR). Documentos temáticos: Objetivos de Desenvolvimento Sustentável. Brasília: ONUBR, 2018. 115p.

PEIXOTO, M.; PINTO, H. S. Desperdício de Alimentos: questões socioambientais, econômicas e regulatórias. Brasília: Núcleo de Estudos e Pesquisas/CONLEG/ Senado, 2016. Boletim Legislativo $n=0$ 41, de 2016. 14p.

PIMENTA, A.S.; GOMES, A.M. Alimentação saudável: Sustentabilidade e Redução do desperdício Alimentar nos hospitais. Hotelaria \& Saúde, n.91, p.12-14, 2012.

PORPINO, G.; LOURENÇO, C.E.; ARAÚJO, C.M.; BASTOS, A. Intercâmbio Brasil União Europeia sobre desperdício de alimentos. Relatório final de pesquisa.
Brasília: Diálogos Setoriais União Europeia Brasil. 2018.

POSPISCHEK, V.S.; SPINELLI, M.G.N.; MATIAS, A.C.G. Avaliação de ações de sustentabilidade ambiental em restaurantes comerciais localizados no município de São Paulo. Demetra, v.9, n.2, p.595-611, 2014.

RODRIGUES, L.P.F.; ZANETI, I.C.B.B.; LARANJEIRA, N.P. Sustentabilidade, segurança alimentar e gestão ambiental para a promoção da qualidade de vida. Participação, n.19, 2012.

ROOS, A.; BECKER, E.L.S. Educação ambiental e Sustentabilidade. Revista Eletrônica em Gestão, Educação e Tecnologia Ambiental, v.5, n.5, p.857-866, 2012.

SANTANA, A.F., OLIVEIRA, L.F. Aproveitamento da casca de melancia (curcubita citrullus, shrad) na produção artesanal de doces alternativos. Revista Alimentos e Nutrição, v.16, n.4, p. 363-368, 2005

SANTOS, M.H.O. Desperdício de alimentos e sua interferência no meio ambiente. Instituto Construir e Conhecer, n.5, 2008.

SAURIM, I.M.L.; BASSO, C. Avaliação de desperdício de alimentos de bufê em restaurante comercial em Santa Maria, RS. Revista Eletrônica Disciplinarum Scientia, v.9, n.1, p.115-120, 2008.

SILVÉRIO, G.A.; OLTRAMARI, K. Desperdício de alimentos em Unidades de Alimentação e Nutrição Brasileiras. Âmbiência Guarapuava, v.10, n.1, p.125133, 2014.

SPINELLI, M.G.N. Gestão adequada dos serviços pode gerar aumento da deficiência operacional e diminuir despesas, beneficiando o meio ambiente. Revista Nutri do Conselho Regional de Nutricionaistas CRN $3^{\circ}$ região, 2009. 
TEIXEIRA, S.; OLIVEIRA, Z.M.C.; REGO, J.C.; BISCONTINI, T.M.B. Administração aplicada às unidades de alimentação e nutrição. Rio de Janeiro: Atheneu, 2006. 219 p.

TEIXEIRA, F.; NUNES, G.; ANTONOVICZ, S.; SILVA, C.C. Principais fatores associados aos índices de desperdício em unidades de alimentação e nutrição: uma revisão integrativa. Saúde em Revista, v. 17, n. 47, p. 43-50, 2017.

VALLE, D.P.; MARQUES, V.S. Biossegurança em unidade de alimentação e nutrição. 1. ed. São Paulo: Atheneu, 2006. $98 \mathrm{p}$.

VIEIROS, M.B.; PROENÇA, R.P.D.C. Avaliação Qualitativa das Preparações do Cardápio em uma Unidade de Alimentação e Nutrição - Método AQPC. Revista Nutrição em Pauta, v.11, n.62, 2010.

VIERA, V.B.; TAMBARA, T.T.; BUZATTI, N.B.; BARBOSA, A.L.R.; LOPES, M.R.; MIRON, V.R.; SACCOL, A.L.F. Análise Sensorial de Sucos Elaborados com Aproveitamento Integral de alimentos. $3^{\circ}$ Jornada Interdisciplinar em Saúde, 2010.

ZANDONADI, H.S.; MAURÍCIO, A.A. Avaliação do índice de resto-ingesta, de refeições consumidas por trabalhadores da construção civil no município de Cuiabá, MT. Higiene Alimentar, v.26, n. 207, p.64-70, 2012. 